\title{
Angiosarcoma of the Heart: Case Report
}

Sofie Dhaeyer ${ }^{1}$, Chirik Wah Lau ${ }^{1}$, Vanessa Meert ${ }^{2}$, Jan Leeman ${ }^{1}$, Martin Penicka ${ }^{1}$, Marc Vanderheyden ${ }^{1 *}$

${ }^{1}$ Heart Failure Unit, Cardiovascular Research Centre, OLV Hospital Aalst, Moorselbaan 164, B-9300 Aalst, Belgium.

${ }^{2}$ Department of Pathology, OLV Hospital Aalst, Moorselbaan 164, B-9300 Aalst, Belgium.

*Corresponding Author: Marc Vanderheyden, Heart Failure Unit, Cardiovascular Research Centre, OLV Hospital Aalst, Moorselbaan 164, B9300 Aalst, Belgium.

\section{Received Date: October 30, 2020; Accepted Date: November 16, 2020; Published Date: November 20, 2020}

Citation: Sofie Dhaeyer., Chirik W, Lau., Vanessa Meert., Jan Leeman., Martin Penicka., Marc Vanderheyden., (2020) Angiosarcoma of the Heart: Case Report. J. Clinical Cardiology and Cardiovascular Interventions, 3(11); Doi:10.31579/2641-0419/100

Copyright: (C) 2020 Marc Vanderheyden, This is an open-access article distributed under the terms of the Creative Commons Attribution License, which permits unrestricted use, distribution, and reproduction in any medium, provided the original author and source are credited.

\begin{abstract}
Cardiac angiosarcomas are rare malignant tumors, predominantly affecting the right heart with poor survival outcomes. The current mainstay of treatment consists of surgery with or without chemotherapy, but often yields limited results with local relapse or metastatic recurrence.

This case report describes 2 patients with primary angiosarcoma located in the right atrium. One patient received neoadjuvant and adjuvant chemotherapy; both were scheduled for surgical resection. The course of disease is described followed by a comprehensive review of the literature.
\end{abstract}

Key Words: angiosarcoma; heart; primary cardiac tumors

\section{Introduction}

Primary cardiac tumors are extremely rare and are more frequently benign than malignant. [1] When malignant, the majority of them are lymphomas or sarcomas. [2] The Armed Forces Institute of Pathology Files reported 1 sarcoma among 533 primary cardiac tumors [3] and Burke et al [4] illustrated that of the 75 sarcomas diagnosed, 26 were angiosarcomas $(35 \%)$. This case report describes 2 patients with primary angiosarcoma located in the right atrium. One patient received neo-adjuvant and adjuvant chemotherapy; both underwent surgical resection. The course of the disease is described followed by a comprehensive review of the literature.

\section{Case presentation}

\section{Case 1}
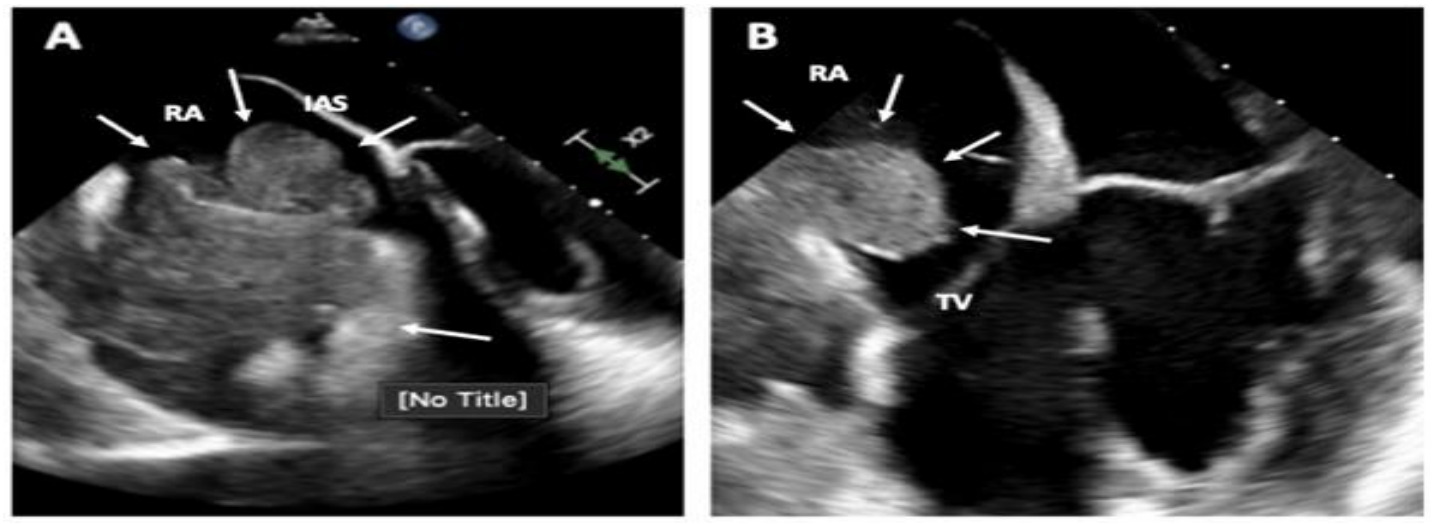

Figure 1: Four chamber transoesophageal image, showing echocardiographic regression of a mass originating from the wall of the right atrium, at time of diagnosis(Panel A) and following chemotherapy(Panel B) with paclitaxel .

A 68-year old Caucasian man with no medical history was admitted with complaints of progressive dyspnea, anorexia, abdominal pain and headache for the past 6 months. Six months earlier when symptoms started, a tentative diagnosis of polymyalgia was made and methylprednisolone was initiated with initial good response. On admission he presented with signs of right heart failure with anasarca edema, ascites and elevated central venous pressure. Abdominal ultrasound confirmed the presence of ascites, however without apparent signs of liver disease. Unexpectedly it also demonstrated an intracardiac mass which, on transoesophageal echocardiography (TOE), revealed to be a floating multilocular mass originating from the lateral wall of the right atrium responsible for a vena cava syndrome with obstruction of the tricuspid valve (Figure 1A). 


\section{IAS indicates interatrial septum, TV tricupid valve, $R A$ right atrium.}

Interestingly this mass was not present on a transthoracic echocardiogram (TTE) performed 9 months earlier. On cardiac CT and MRI, the intracardiac mass with a maximal diameter of $11.5 \mathrm{~cm}$ was highly vascularized. Furthermore, pericardial invasion, encasement of the right coronary artery and a large subcarinal lymph node were seen. On PETCT, high fluoro-18-deoxyglucose (FDG) uptake was observed at the level of the right atrium and the area around as well as in two spots on the left oropharynx and left humeral head (Figure 2).
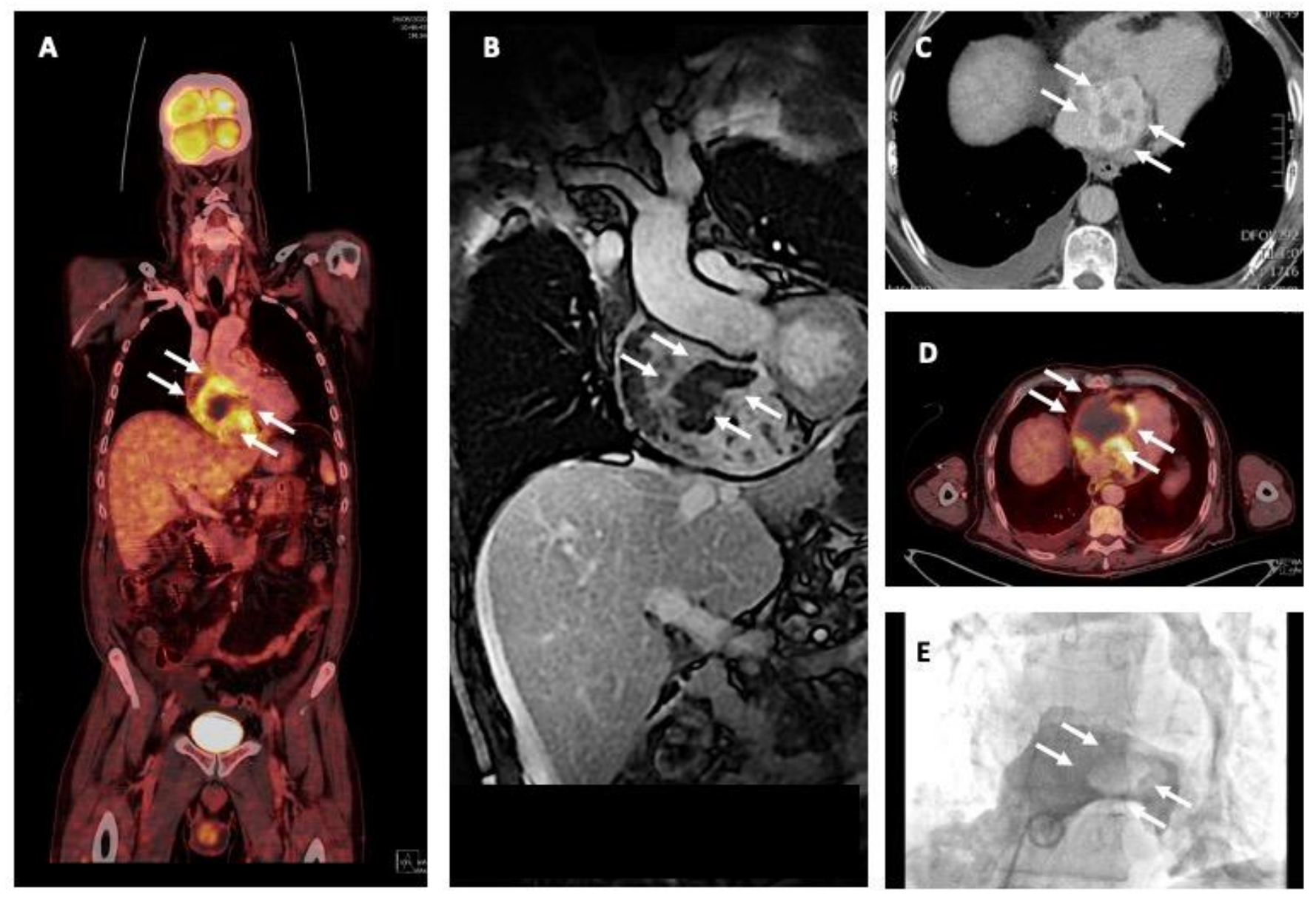

Figure 2: PET-CT (panel A +D), CT (panel C) MRI (panel B) and RV angiogram (panel E). A floating multilocular mass (arrow) originating from the lateral wall of the right atrium partly obstructing the tricuspid valve with a maximal diameter of 11,5 cm and high FDG uptake was observed

These 2 hot spots were related to an inclusion cyst and arthrosis of the shoulder. Arguments for metastasis were absent. Histological and immunohistochemical analysis of tissue obtained by a transfemoral biopsy showed infiltrative blood vessels with atypical cells staining positive for ERG, CD31 with a Ki-67 proliferation index of 50\%, compatible with the diagnosis of angiosarcoma (Figure 3). 

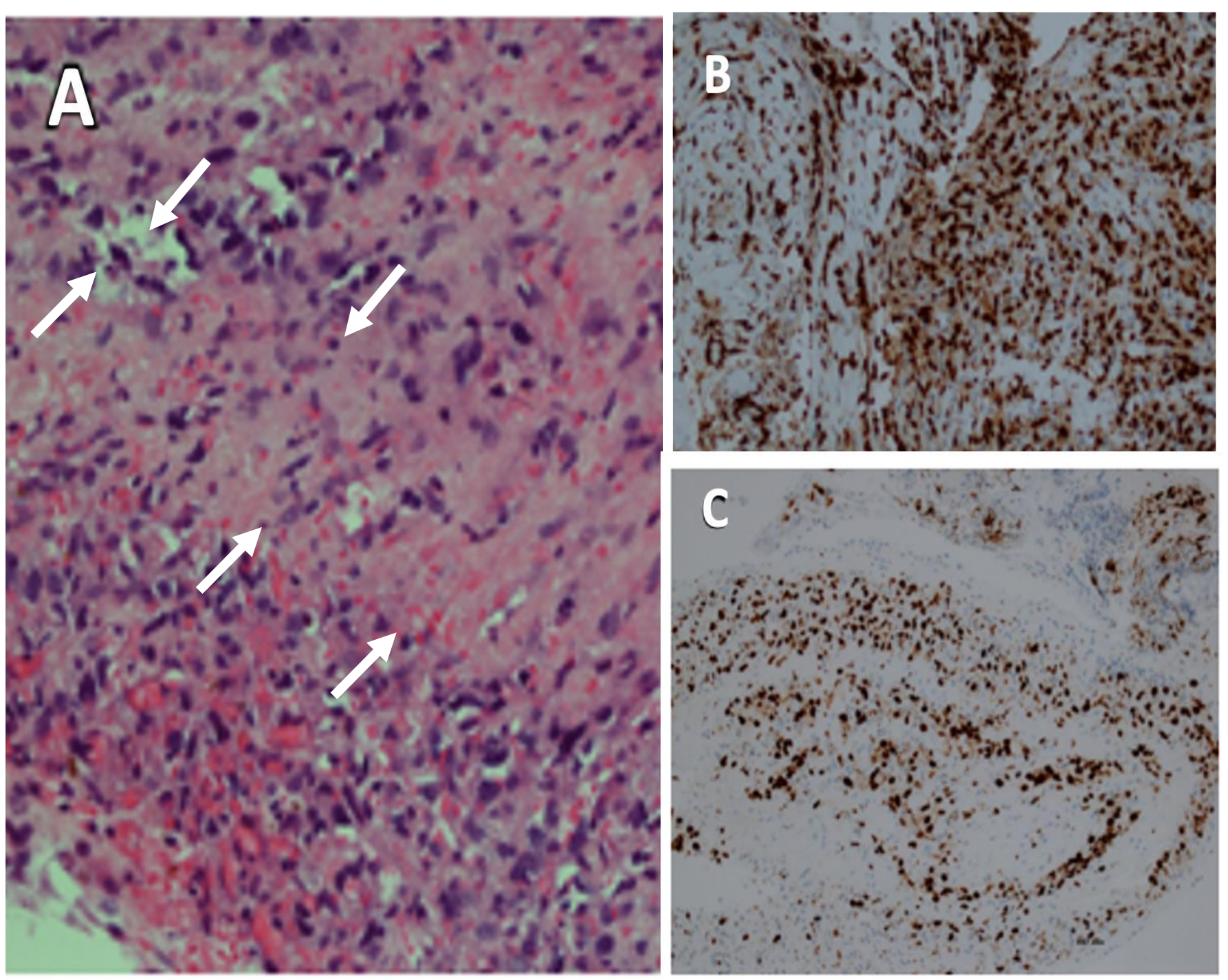

Figure 3: H\&E stain (panel A), ERG stain (panel B), Ki67 stain (panel C). Panel A: 200 times magnification of H\&E stain showing malignant endothelial cells (white arrows). Panel B: 100 times magnification of IHC staining for ERG, confirming vascular differentiation. Pancel C: 100 times magnification of IHC for Ki67 showing a proliferation index of $50 \%$.

$H \& E=$ Hematoxyline and Eosin $; I H C=$ immunohistochemical $; E R G=$ ETS-related gene

The clinical staging of the tumor was cT2b cN1 M0. To downsize the tumor and facilitate surgical resection neoadjuvant chemotherapy with 3weeks of treatment with paclitaxel followed by 1 week of rest for 12 cycles was initiated. Re-evaluation after 6 cycles showed good clinical response with significant echocardiographic regression (Figure 1B). Surgery was proposed and will be planned after completion of the chemotherapy (cfr discussion)

\section{Case 2}

An 88-year old Caucasian man with a past history of mitral valve annuloplasty (Carpentier-Edwards Physio II ring) was admitted with complaints of general discomfort, anorexia and dyspnea. He had been treated with antibiotics for a bronchial infection, though without any clinical improvement. On presentation, tachycardia (atrial fibrillation) and low oxygen saturations were noted with signs of left sided congestive heart failure with bilateral pleural effusion on chest X-ray and an elevated serum NT-proBNP. He was admitted with a tentative diagnosis of acute decompensated heart failure triggered by recent respiratory infection and new onset atrial fibrillation.

Transthoracic echocardiography revealed a right atrial mass with a diameter of $7.3 \mathrm{~cm}$ (Figure 4). 

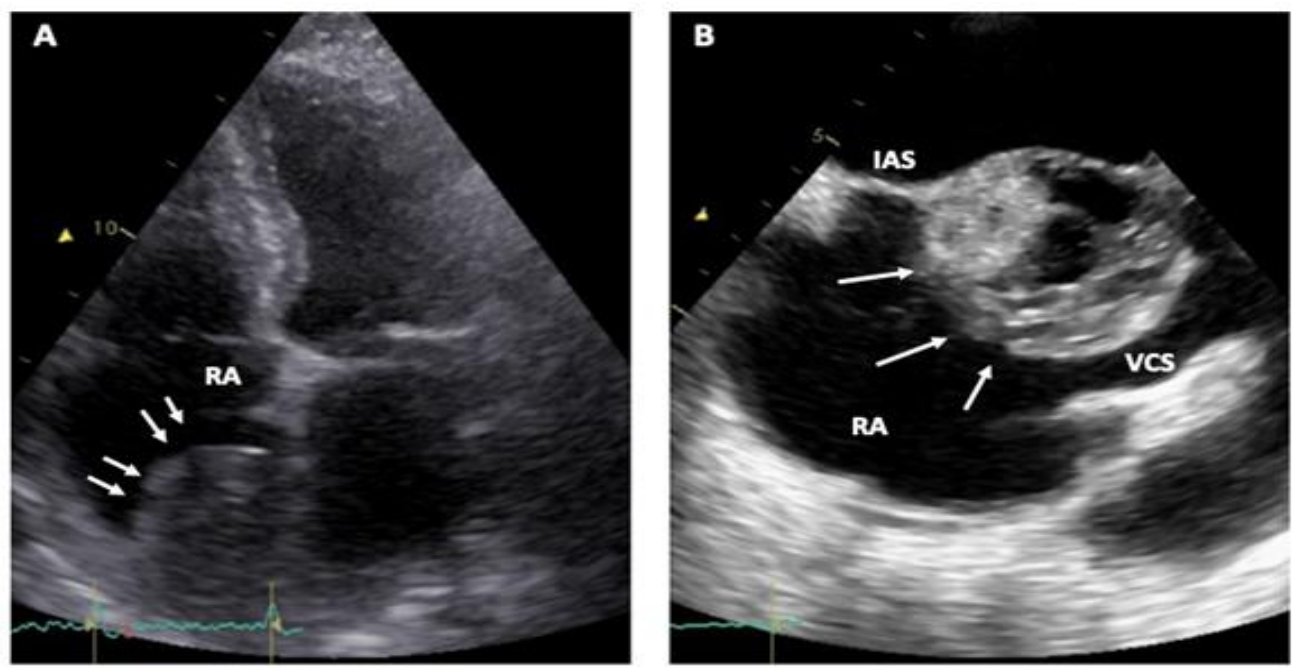

Figure 4: Transthoracic four chamber (panel A) and transoesophageal bicaval(panelB) image showing a mass(arrow) obstructing the superior vena cava. IAS indicates interatrial septum, VCS vena cava superior, RA right atrium.

Similar to the previous case a TTE obtained thirteen months before showed no atrial mass. On MRI the mass originated from the interatrial

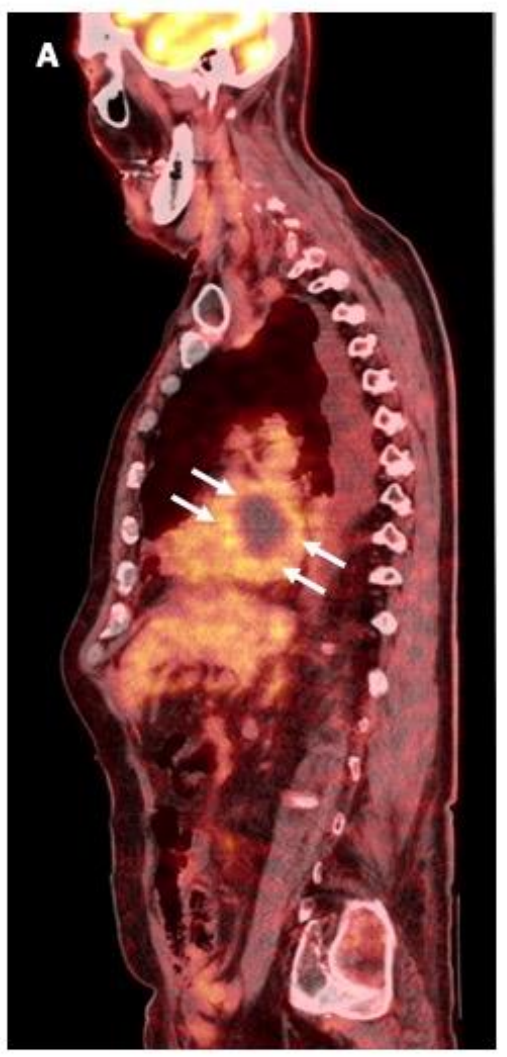

septum of the right atrium with a signal intensity suggestive of a myxoma, although a sarcoma could not be ruled out (Figure 5).
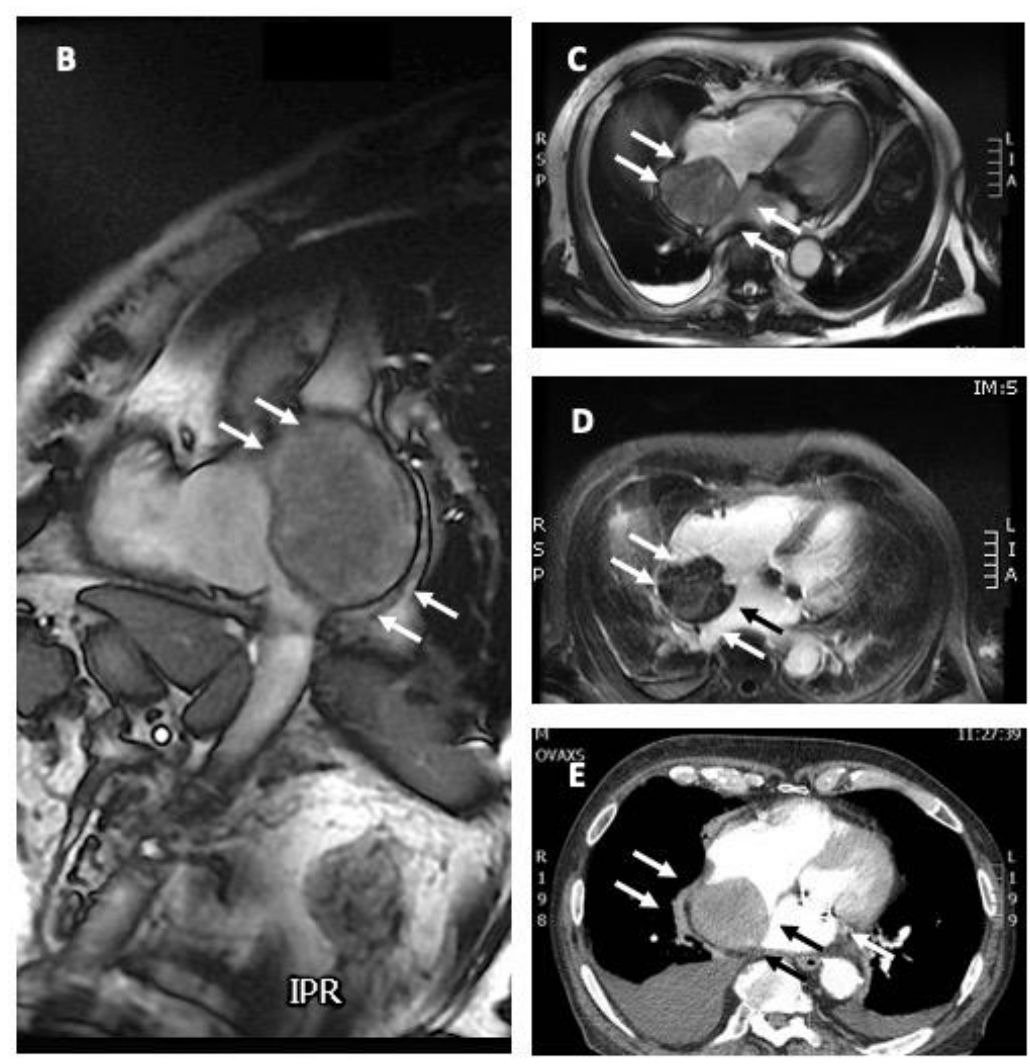

Figure 5: PET-CT (panel A), CT (panel E) MRI (panel B, C, D). A right atrial mass (arrow) with a diameter of $7.3 \mathrm{~cm}$, originating from the interatrial septum and FDG-uptake.

Additional PET-CT showed low FDG uptake of the atrial mass, metastatic disease could not be detected (Figure 5). Under extracorporeal circulation the mass was resected, and the interatrial septum was reconstructed with a bovine pericardium patch. Anatomopathological examination revealed the mass to be an angiosarcoma, with positive staining for CD31 and $\mathrm{Ki}$ 67(Figure 6). 

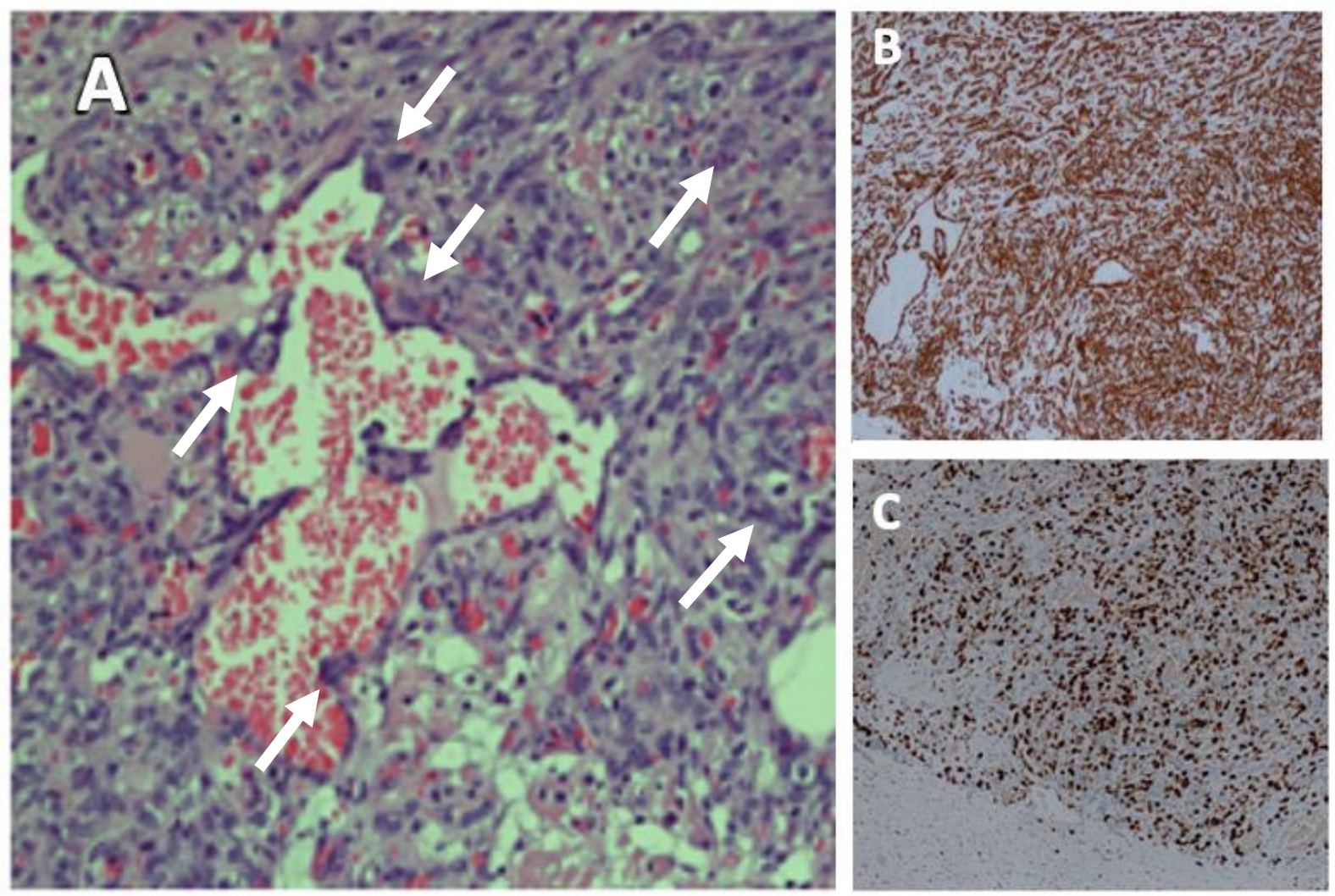

Figure 6: H\&E stain (panel A), CD31 stain (panel B), Ki67 stain (panel C). Panel A: 200 times magnification of H\&E stain showing malignant endothelial cells (white arrows) and slit like blood vessels. Panel B: 100 times magnification of IHC CD31 staining, confirming vascular differentiation. Panel C: 100 times magnification of IHC Ki67 staining showing a proliferation index of $80 \%$.

Although the resection margins were not tumor-free, no adjuvant chemotherapy was given because of patient's advanced age and general condition. Six weeks following surgery he was readmitted because of progressive dyspnea due to a massive right sided pleural effusion. Three liters of fluid were evacuated. Histological examination of the fluid didn't show atypical or malignant cells, nor signs of tumor recurrence. Despite these reassuring findings relapsing pleural effusions occurred, ultimately treated by a thoracoscopic pleurodesis. Four months after surgery the patient presented with cachexia and abdominal ultrasound showed liver metastasis. Considering the advanced age, frailty and expected prognosis, he was referred for palliative care.

\section{Discussion}

Sarcomas, particularly the angiosarcomas, are vascular in origin and typically reside in the right-sided structures of the heart with predilection for the right atrium. [5] They often occur in a young age population, but as observed in the second patient have also been described in any agegroup. Frequent presenting symptoms include dyspnea (44\% of the patients), chest pain (8\%) or shortness of breath, attributed to pleural effusion (28\%). [2]

Cardiac angiosarcoma is defined by endothelial differentiation and characterized by a high proliferation rate, permeating and destructive nature, propensity to metastasize, and poor prognosis with patients succumbing to disease within a few months of diagnosis. Molecular aberrations detected in cardiac angiosarcoma often involve $K D R$ and $K I T$ and homozygous deletion of $C D K N 2 A$. The presence of somatic mutations in angiogenic signaling pathways, provides a rationale for investigating targeted therapies. [5]
As cardiac angiosarcomas are extremely rare, evidence-based therapeutic guidelines are lacking and most therapeutic strategies are based on retrospective studies and case reports. Ultimately the cornerstone of every treatment plan with curative intent remains complete R0 surgical resection, defined as macro- and microscopical tumor free resection margins. Whenever possible, this results in a median survival of 17-53.5 months vs 6-9.1 months for those in whom resection is incomplete. [6,7] As the tumor often grows into critical structures such as the tricuspid valve, not only the location but also its relationship with the surrounding structures determines the type of surgery. In this regard two identical cases to our first one with encasement of the right coronary artery and tumor growth into the tricuspid valve apparatus have been described where tumor, RCA and tricuspid valve were completely resected followed by replacement of the tricuspid valve and reconstruction of the right atrioventricular wall with bovine pericardium. Though grafting of the RCA happened only in one of the two cases, both patients had a good right ventricular function postoperatively. [8,9] Although initial successful, the first patient died of metastasis 10 months after the surgery. The second patient survived 12 months without metastasis. No further data were registered. Hirose $\mathrm{K}$ et al. described a similar case with complete resection of an angiosarcoma invading the right ventricular wall close to the tricuspid valve. Nevertheless, also this patient died three months later due to pulmonary metastasis in absence of local recurrence. [10]

In those with tumors located in the left atrium, heart transplantation can be an option. However even for these patients the prognosis remains poor with a median survival time of 9 months after heart transplantation, one month longer than the survival reported after palliative therapies. [11,12] 
Other therapeutic modalities such as chemo- and radiotherapy have been explored with intention to downsize the tumor and increasing the odds for a complete surgical resection, as adjuvant treatment in case of metastasis or in a palliative setting. Anthracyclines, ifosfamide and paclitaxel are the chemotherapeutic agents of choice. [13] Large randomized trials evaluating the impact of chemotherapy on survival are scarce. The biggest trial evaluating the preoperative treatment with doxorubicin/ifosfamide to downsize the tumor, demonstrated a significant higher overall survival of 20 months compared to 9 months for those who only underwent surgery. [14] Similar outcome and effects on tumor reduction with less adverse effects and better tolerance have been reported for monotherapy with doxorubicin. [15] Also the retrospective ESPERO trial demonstrated a survival benefit for the group of patients who received neoadjuvant chemotherapy with either ifosfamide/adriamycine or gemcitabine/docetaxel. [6]

In patients with metastatic disease, similar chemotherapeutical agents have been used in combination with surgical resection. Experimental as well as clinical studies with combination therapy with doxorubicin/cyclophosphamide/vincristine as well as paclitaxel with or without doxorubicin have demonstrated a positive impact on the metastatic spread of the tumor and survival. [16-19]

Recently the role of immunotherapy has been investigated. It has been shown that patients receiving combination therapy with doxorubicin and olaratumab (26.5 months) as well as patients on a combination therapy with doxorubicin and olaratumab (14.3months) had a better prognosis compared to placebo/standard therapy. [19,20]

As KDR and VEGDR2, both markers of angiogenesis and vasculogenesis, are highly expressed in angiosarcomas and somatic mutations in angiogenic signaling pathways are often observed [5], molecules inhibiting vasculogenesis such as initinib, lenvatinib, pazopanib, regorafenib, vatalanib, and tivozanib are good candidates for targeted therapy. [22] In this regard, the PALETTE trial, comparing pazopanib to placebo in soft-tissue angiosarcoma, showed significant benefit for progression-free survival of pazopanib. [23]

Finally, also radiation therapy combined with surgery and/or chemotherapy can be an option. However, high radiation doses with direct cardiotoxic effect are often needed due to technical challenges related to the movement of the tumor. Newer technologies such as breath-hold sequences, intensity-modulated radiotherapy tracing and fourdimensional tumor analysis are under development in an attempt to overcome these challenges. [24]

\section{Conclusions}

Cardiac angiosarcomas are rare neoplasms, predominantly affecting the right heart. With scarce evidence from literature the current mainstay of treatment is complex an consists of surgery with or without chemotherapy. Outcome remains very poor. Therefore, there is an urgent need of more adequate tools for early diagnosis and more effective treatment.

\section{References}

1. Silverman NA. Primary cardiac tumors. Ann Surg 1980; 191:127-138.

2. Tazelaar HD, Locke TJ, McGregor CGA. Pathology of surgically excised primary cardiac tumors. Mayo clinic Proc 1992; 67: 957-965.

3. McAllister HA, Fenoglio JJ. Tumors of the cardiovascular system. In: Atlas of Tumor Pathology, Fascicle 15, series 2. Washington DC: Armed Forces Institute of Pathology 1978.

4. Burke AP, Cowan D, Virmani R. Primary sarcomas of the heart. Cancer 1992; 69(2):387-395.
5. Urbini M, Astolfi A, Indio V, et al. Genetic aberrations and molecular biology of cardiac sarcoma. Ther Adv Med Oncol 2020;12:1758835920918492

6. Abu Saleh WK, Ramlawi B, Shapira OM, Al Jabbari O, Ravi V, Benjamin R, et al. Improved Outcomes With the Evolution of a Neoadjuvant Chemotherapy Approach to Right Heart Sarcoma. Annals of Thoracic Surgery. 2017;104(1):90-96.

7. Blackmon SH, Reardon MJ. Surgical treatment of primary cardiac sarcomas. Texas Heart Institute Journal. 2009;36(5):451452 .

8. Kwon Y, Park SJ, Kim HJ, Kim JB. Complete Resection of Cardiac Angiosarcoma Invading Right Heart and Right Coronary Artery. The Annals of thoracic surgery. 2020.

9. Donmez AA, Cekmecelioglu D, Adademir T, Yilmaz E, Alp HM. Primary Cardiac Angiosarcoma with Rare Presenting Feature and Successful Surgical Treatment. Braz J Cardiovasc Surg [Internet]. 2018 [geciteerd 7 september 2020];33(5). Beschikbaar op:

10. Hirose K, Miyazaki S, Morimoto R, Maruyama M, Nishiyama $\mathrm{H}$, Takagi A, et al. A case of primary cardiac angiosarcoma accompanied with severe aortic stenosis. European Heart Journal Cardiovascular Imaging. 2019;20:i828.

11. Überfuhr P, Meiser B, Fuchs A, Schulze C, Reichenspurner H, Falk M, et al. Heart transplantation: An approach to treating primary cardiac sarcoma? Journal of Heart and Lung Transplantation. 2002;21(10):1135-1139.

12. Li H, Yang S, Chen H, Yang Z, Hong T, Hou Y, et al. Survival after heart transplantation for non-metastatic primary cardiac sarcoma. Journal of cardiothoracic surgery. 2016;11(1):145.

13. Young RJ, Brown NJ, Reed MW, Hughes D, Woll PJ. Angiosarcoma. Lancet Oncol. oktober 2010;11(10):983-991.

14. Ramlawi B, Leja MJ, Abu Saleh WK, Al Jabbari O, Benjamin R, Ravi V, e.a. Surgical Treatment of Primary Cardiac Sarcomas: Review of a Single-Institution Experience. Ann Thorac Surg. februari 2016;101(2):698-702.

15. Maurel J, López-Pousa A, de las Peñas R, Fra J, Martín J, Cruz J, e.a. Efficacy of Sequential High-Dose Doxorubicin and Ifosfamide Compared With Standard-Dose Doxorubicin in Patients With Advanced Soft Tissue Sarcoma: An Open-Label Randomized Phase II Study of the Spanish Group for Research on Sarcomas. J Clin Oncol. 10 april 2009;27(11):1893-1898.

16. Zhang ZY, Cheng YJ, Gong XL, Ge YP, Bai CM, Wang XJ, e.a. [Characteristics and outcomes of primary angiosarcoma]. Zhonghua Zhong Liu Za Zhi. 23 september 2019;41(9):693-697.

17. Truong PT, Jones SO, Martens B, Alexander C, Paquette M, Joe $\mathrm{H}$, e.a. Treatment and outcomes in adult patients with primary cardiac sarcoma: the British Columbia Cancer Agency experience. Ann Surg Oncol. december 2009;16(12):3358-3365.

18. Ahn H, Kim HK, Yoo KH, Kim YS, Lee SJ. Primary cardiac sarcoma: A retrospective study in two Korean tertiary centers. Ann Oncol. september 2017;28:v527.

19. Braham W, Pisani A, Poitier B, Sannier A. Primary cardiac synovial sarcoma: an asymptomatic patient 8 years after the primary surgery. Interact Cardiovasc Thorac Surg. 1 september 2018;27(3):471-473.

20. Tap WD, Jones RL, Van Tine BA, Chmielowski B, Elias AD, Adkins D, e.a. Olaratumab and doxorubicin versus doxorubicin alone for treatment of soft-tissue sarcoma: an open-label phase $1 \mathrm{~b}$ and randomised phase 2 trial. The Lancet. juli 2016;388(10043):488-497.

21. D'Angelo SP, Mahoney MR, Van Tine BA, Atkins J, Milhem MM, Jahagirdar BN, e.a. Nivolumab with or without ipilimumab treatment for metastatic sarcoma (Alliance A091401): two open- 
label, non-comparative, randomised, phase 2 trials. Lancet Oncol. 2018;19(3):416-426.

22. Zhrebker L, Cherni I, Gross LM, Hinshelwood MM, Reese M, Aldrich J, e.a. Case report: whole exome sequencing of primary cardiac angiosarcoma highlights potential for targeted therapies. BMC Cancer. december 2017;17(1):17.

23. Schur S, Hamacher R, Brodowicz T. Pazopanib in Primary Cardiac Angiosarcoma of the Right Atrium: A Case Report. Case Rep Oncol. 27 juni 2016;9(2):363-337.

24. Look Hong NJ, Pandalai PK, Hornick JL, Shekar PS, Harmon DC, Chen Y-L, e.a. Cardiac Angiosarcoma Management and Outcomes: 20-Year Single-institution Experience. Ann Surg Oncol. augustus 2012;19(8):2707-2715.

cC)

This work is licensed under Creative

Commons Attribution 4.0 License

To Submit Your Article Click Here: Submit Article

DOI: $10.31579 / 2641-0419 / 100$
Ready to submit your research? Choose Auctores and benefit from:

* fast, convenient online submission

* rigorous peer review by experienced research in your field

* rapid publication on acceptance

* authors retain copyrights

* unique DOI for all articles

* immediate, unrestricted online access

At Auctores, research is always in progress.

Learn more www.auctoresonline.org/journals/clinical-cardiology-andcardiovascular-interventions- 